\title{
PHYSIOTHERAPY IN COMBINATION OF KARATE TECHNIQUES IN A CHILD WITH KABUKI SYNDROME - CASE PRESENTATION
}

\author{
Nezabravka Gencheva ${ }^{1}$, Yordan Georgiev ${ }^{2}$ \\ ${ }^{1}$ National Sports Academy „Vassil Levski“, ${ }^{2}$ DKC „Sveta Marina“, Sofia, Bulgaria
}

\begin{abstract}
Kabuki syndrome is a rare genetic disorder that affects one in 32,000 newborns. It is characterized by multiple abnormalities including distinctive facial features, growth delays, varying degrees of intellectual disability, heart abnormalities, muscle hypotension that could affect the motor functions, etc.

The aim of the study is to evaluate the changes in the motor functions in a child with Kabuki Syndrome after appropriate physiotherapy treatment in combination with karate techniques.

Methodology. One year physiotherapy course was held in a 14 years old boy with Kabuki syndrome. The changes in the motor function were examined using GMFM. Functional Independence Measure Weefim scale was applied for assessing independence of the child and limp circumference measurement was examined for evaluating the muscle hypotrophy. The physiotherapy and karate trainings were held 6 times per week and aimed to enhance muscle tone and impact on postural muscles. The therapy was also working on improving posture, equilibrium responses and coordination as well as reducing social deficits.

Results. The favorable development of gross motor functions (GMFM) could be seen mostly in child activities during walking, running and jumping. The results show increase in the muscle strength for the examined period. Improved selfservice skills as well as improved social interaction are observed (VeeFim).

Conclusion. The Kabuki syndrome is a rare genetic disorder and no definitive conclusions could be made. The current study represents good practices for PT application in combination of adaptive sports. The results could be used as a basis for further in-depth research.
\end{abstract}

Key words: kabuki syndrome, physiotherapy, karate

\section{INTRODUCTION}

Kabuki syndrome is a rare genetic disease which appears in one of 32,000 babies. The name comes from the resemblance with the make-up of the actors from the most ancient Japanese theatre Kabuki. The disease is characterized with numerous abnormalities including striking facial features, growth retardation, various degree of intellectual difficulties, skeletal abnormalities, heart abnormalities, etc. (Niikawa et al. 1982, Kuroki et al. 1981). The etiology of Kabuki make-up or Niikawa-Kuroki syndrome is not completely clear yet. Most cases are gene mutations. Between 55 and 80 percent of Kabuki syndrome cases are caused by mutations in the KMT2D gene (also known as MLL2). Between 2 and 6 percent of cases of Kabuki syndrome are caused by mutations in the KDM6A gene (Niikawa $\mathrm{N}$ et al.1988). Some people with Kabuki syndrome have no identified KMT2D or KDM6A gene mutation. The cause of the disorder in these individuals is unknown. $\mathrm{X}$ - related or autosome dominant type heredity is accepted. A number of papers report for hereditary defectiveness with Kabuki syndrome (Halal et al.1989). Hannibal M. et al. (2011), who researched 110 families with Kabuki syndrome, found in 81 families (74\%) mutation of the gene MLL2 which coordinates the proteins of the Trithorax group regulating the structure of chromatin and participating in the maintenance of gene expression.

The syndrome has 5 basic characteristics which do not appear in the same patient every time. The facial phenotype is specific and easily recognizable regardless of the ethnic origin. The unusual face is found in $100 \%$ of the patients with this syndrome; abnormal dermatoglyphic model, $93 \%$ skeletal abnormalities, 92\% mental retardation; 92\% postnatal growth deficiency, $83 \%$. The characteristic facial features are: eversion of the lateral third of the lower eyelid (features resembling Kabuki actors, traditional Japanese play), broad, flat nasal tip; large, prominent, or cupped ears; cleft or highly arched palette (Carcione, et al 1991, Niikawa, Kuroki, 1982). Skeletal abnormalities: they may include brachydactyly of the toes, 
webbed fingers are a rarer abnormality (Matsumoto \& Niikawa 2003), spinal column abnormalities, (butterfly vertebrae, sagittal cleft vertebrae and scoliosis), pseudarthrosis clavicle, repetitive dislocation of the patella, hyper joint laxity, etc. (Wada, et al 2012). Dislocations of joints are found in $50 \%$ of the patients with KS. The dislocation of the patella may cause disability and might require a surgery (Rouffiange, 2012, Geneviève, et al 2004) Some of the most often reported sensory problems include the necessity of oral stimulation - including chewing of nonfood products, tangible defense to different sensations and stimuli, panic reactions to certain noises and disgust to fabrics or smells. Dermatological abnormalities: including constantly folded fingers due to the embryonic origin. The mental retardation is mild to moderate. An index from the diagnostics is shortness after birth. The short stature of the baby is one of the main anthropometric characteristics of Kabuki syndrome. It is not yet clear to what extent the hGH deficiency contributes to this characteristic. Although weight and length after birth are usually normal, growth retardation often starts during the first year after birth. There are some surveys which confirm that younger children who undergo a treatment with hGH significantly increase their stature and improve the proportions of the body compared to those who start the treatment at a later age. In this prospective survey 18 genetically proven children with KS (9 females and 9 male) aged between 3.8 and 10.1 years (mean $6.8 \pm 2.1$ years) were treated with recombined hGH (rhGH) over a year (Schott D. et al 2017). Devriendt K et al.(1995) introduced a girl with Kabuki syndrome whose treatment with hGH led to an increase in her stature with annual growth of 3.6 to $11.2 \mathrm{~cm}$. In his research Philip N. et al. (1992) made a conclusion that there were two differences between Japanese patients and those included in that research. The first difference was that two thirds of the patients in the research had a significant neurological dysfunction different from mental retardation. The second difference was that the hypermobility of the joints appeared more often among non-Japanese patients. The authors made a conclusion that the confirmation of these findings required further research.

What is important for us as regards physiotherapy is that different neurological problems exist among people with Kabuki syndrome. The most prevalent is hypotony which may prevent the development of motor functions and nutrition. Other neurological abnormalities include microcephalia and fits. The physiotherapy of Kabuki syndrome should play a central role in rehabilitation of the disease due to the possibilities it offers for improvement of the muscle tone, motoric, balance-coordination abilities of a child, sensory stimulation, functional skills for self-catering, etc. Presently, there is not sufficient amount of literature about the physiotherapeutic approaches in treating Kabuki syndrome. Some publications just mention the necessity of its implementation in the conservative treatment for enhancing the weak muscles and post-surgical treatment of patella dislocation (Geneviève, 2004). People with Kabuki syndrome usually have a low tone - hypotony. A physiotherapist is able to assess and develop a long-term plan for strengthening the muscles and change the functioning of the individual. A child with Kabuki syndrome often feels languid/tired and needs a lot more efforts to perform different activities. By applying appropriate physiotherapeutic programs, one can achieve motor activity very close to the abilities of a healthy child at the same age. The physiotherapy is aimed at stimulation of the motor development of a child and during the breastfeeding period and first months it is aimed at learning how to turn around independently, how to craw and perhaps walk. This is especially important for people with Kabuki syndrome because apart from hypotony they may have ligament laxity which might lead to joint impairment. There might be a complication such as dislocation of the knee cap. That is why special exercises should be applied when children grow up so that their knees get more stable and the dislocation of the knee cap be avoided. The growing-up girls are more apt to knee cap dislocation during puberty due to a change (and possibly increase) in weight distribution.

The aim of the study is to evaluate the changes in the motor functions in a child with Kabuki Syndrome after appropriate physiotherapy treatment in combination with karate techniques.

\section{METHODOLOGY}

We report a case of a 14-year-old white boy with genetically proven KS type I, showing a mutation of the KMT2D gene. (another term - MLL2). Gene KMT2D is located in chromosome 12q13.12 The patient was a second born child, born with Sectio Ceasareae with urinary tract infection right after birth with weight of $3000 \mathrm{gr}$. He had mental retardation resulting in developmental disabilities. No other options were found through sequence analysis of the remains of the gene KMT2D or KDM6A. 
This result gives a molecule confirmation of the diagnosis for Kabuki syndrome. The research on the cardiovascular activity at the beginning of the treatment showed a lack of cyanosis and cardiovascular insufficiency (CI), symmetric pulse of the four limbs, well filled, rhythmical cardiovascular activity, clear tones, holosystolic noise in the left sternal edge. Our child with Kabuki syndrome (KS) is characterized by typical facial features (long palpebral fissures with eversion of the lateral third of the lower eyelid; arched and broad eyebrows; large, prominent, or cupped ears, etc.).Our observations of the boy with Kabuki syndrome lasted over a period of 1 year The parents were asked to grant an informed permission for the experimental methods and were preliminarily explained the organization of the rehabilitation process. We suggested combining the physiotherapy with karate techniques. The child was consulted by medical specialists who also gave permission for the experimental methods. We tested some daily motor activities in advance, such as: walking, running, squatting, jumping, some self-catering activities, catching with hands, throwing.

Research of the motor functions. We established the following motor difficulties: walking - clumsy, with broad support; running - unsteady and irregular; jumping - with straightened legs, cannot jump on one foot; dressing up - ties his shoes in a knot, puts his jacket on inside out, can fasten a zip but cannot do up and undo buttons; squatting - on toes and hand support; catching - with both hands and the object leans against his chest, if it is away from the chest, he drops it; throwing - only underhand with both hands, he cannot make a chest pass. On the basis of this initial testing we decided to apply the following evaluation tests: Gross Motor Function Measure (GMFM 66) (Russell et al, 2002). GMFM is an observational clinical tool designed to evaluate change in gross motor function in children with cerebral palsy aged between 5 months to 16 years, but we decided to apply the instrument in our research because individuals with Kabuki syndrome lag behind in their gross motoric. WeeFim The FIM ${ }^{\mathrm{rm}}$ instrument comprises 18 items, each of which is assessed against a seven point ordinal scale, where the higher the score for an item, the more independently the patient is able to perform the tasks assessed by that item. Total scores range from 18 to 126. (Slomine, 2011) Centimetric through measurement of Bicep Circumference in contraction $(\mathrm{cm}$.$) and Thigh Circumference (\mathrm{cm}$. of to establish muscle hypertrophy

\section{Physiotherapy}

We decided to substitute part of the physiotherapeutic sessions with karate trainings. We performed 3 karate sessions and 3 physiotherapy sessions three times a week over a period of one year. After the functional diagnostics the child performed analytical exercises for stimulation of all muscle groups of upper and lower limbs, neck and torso which were aimed at increasing the muscle tone and influence the postural muscles improving the posture and balance reactions, muscle stretching exercises (Figures 3-4) breathing exercises, games for stimulation of psycho-motoric, etc. The special karate exercises were aimed at achieving correct positions for performance of the techniques.

\section{Karate Exercises}

Karate is a good option for ataxia patients for impact on the equiaxial system and for children with Kabuki syndrome. The child is bare feet for a better stimulation and improvement of proprioception; achieving a good initial position is a basis for development of movements.

Fudu datchi - the feet are shoulder width apart, at a 45-degree angle. The aim of this position is the maintenance of a proper body posture. Zenkutsu dachi - one leg is forward, bent at the knee. The body is straight, and the rear leg is extended at the knee and coxofemoral joint. The feet are twice shoulder width apart, the front foot points forward, and the rear foot slightly sideward. The aim of the position is improvement of the balance and body coordination and training muscles in isometric regime. Morote shodan ski - a hit with both hands in the stomach area. The aim of the position is improvement of the coordination hand-eye. Shodan $s k i-a$ hit with one hand in the stomach area. The aim of the position is improvement in the accuracy and agility and enhancing the extensor groups of muscles in the arm. Jodan uke - blocking of punch directed to the head, the defender blocks with the forearm. The aim of the position is improvement of coordination eye-hand, training of the muscles in isometric and isotonic regime. Gedan barai blocking of punch directed to the groin, the defender performs it with the forearm. The aim of the position is improvement in the speed and increase in the muscle power. Hiza geri - a hit with a knee. The aim of the position is improvement of balance and coordination, strengthening the muscles in the 
thighs. Kin teki geri - a hit with the foot directed to the groin. The aim of the position is improvement in balance and coordination, strengthening the muscles in the whole lower limb.

\section{RESULTS}

The results from implementation of GMFM at the beginning and at the end of the treatment showed that there was some growth in position 2 sitting po- sition, and the tempo of the growth of these changes was satisfactory -3 . There were slightly expressed positive changes in crawling and kneeling. The bigger growth 11 points was observed in the position related to walking and running. There was no change in the other two researched positions. The total evaluation at the end of the therapeutic course was 64 , $36 \%$ of the maximum one. The total growth in the gross motoric was only $3 \%$. ( Figure 6 )

\section{GMFM}

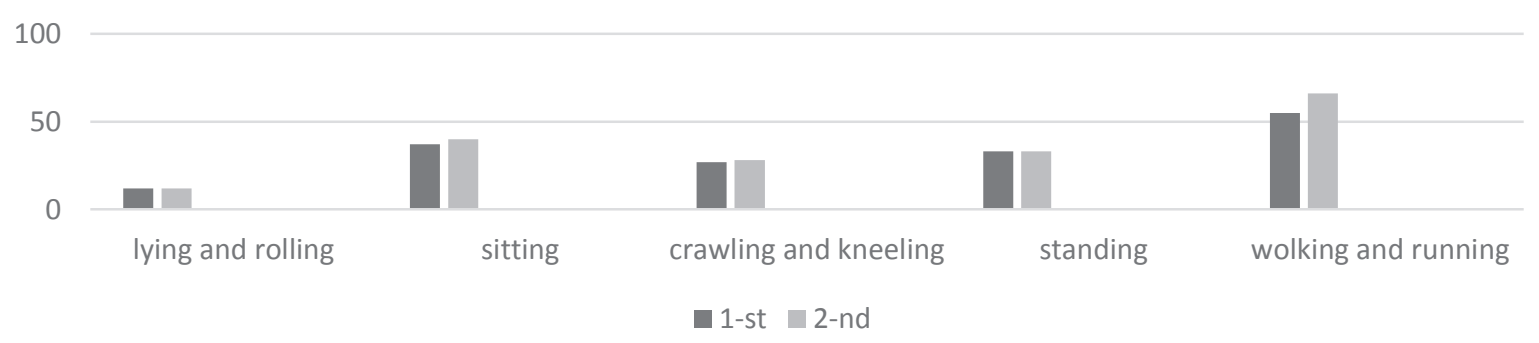

Figure 1. Changes in GMFM

When analyzing these data, we consider that there is a beneficial development in the gross motor functions in the activities which the child performs while sitting, walking or running. The changes in these testing positions are related to the efficiency of implementation and learning the karate techniques which have applied character and influence the postural reactivity of the child with Kabuki syndrome. Regardless of the yearly implementation of physiotherapy and karate techniques, we could see that the gross motoric was hard to influence and a good motor control is difficult to be built which further increases the need of regular physiotherapy activities and different alternative motor activities.

After the final testing, in the general condition of the child there were some improved skills for self-catering in the process of eating, washing, and bathing (VeeFim). He went up and down stairs independently, there was some improvement in the social interaction, as well as in taking his own decisions. As regards expression, social interaction with other people, taking decisions and memory, the child still needed some external minimal stimulation and support on behalf of his parents, relatives and teachers After the end of the treatment, he still had some difficulties dressing up and taking off clothes, and in sphincter control which directed us towards developing and implementing some specialized exercises aimed at achieving full independence, which is related to the weak motor control established in gross motor function. (Figure 2).

\section{VeeFim}

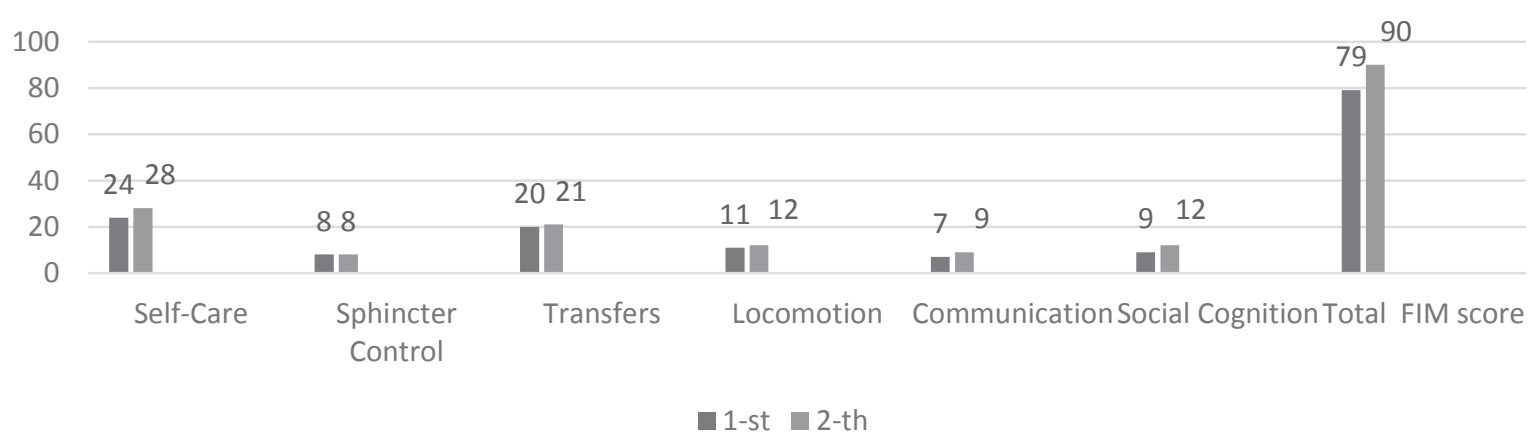

Figure 2. Changes in VeeFim

At the end of the therapeutic course the child showed a better understanding to instructions 
and requirements. As a whole, the total number of points increased at the end of the research period in comparison to its beginning

The data from the measurement of circumferences of armpit at rest and bent and thigh circumference showed there was a slight increase at the end of the research period which meant an improvement in the muscle tone and building up muscles from the regular karate trainings and strength exercises $\mathrm{Bi}$ cep Circumference contraction $(\mathrm{cm})$ from 30 to 31 sm and Thigh Circumference from 50 to $51 \mathrm{sm}$.

\section{DISCUSSION}

The minimal changes observed in GMFM of the child with Kabuki syndrome reveal the great necessity of regular physiotherapy activities and different alternative motor activities. The regular execution of analytical physical exercises and karate techniques helped the child to perform functional daily movements properly and independently or with a little help. A regulation of the muscle tone is made through execution of different karate techniques while walking. Lots of the techniques are performed in one place initially, then after their learning they are performed while walking. In this position, while stepping forward slowly and carefully some corrections of the spinal column are possible. When summing up the movement of the limbs, one can achieve correction in the pathological motor models and posture through redistribution of the load, therefore, the movement of the limbs can be used for straightening the torso and coordination. The execution of more than one technique while walking is related to an increase in the motor stimulus and perfection of coordination and balance.

The sensory defense is a common problem in the children with Kabuki syndrome. The sensory defense can prevent children from desire to move and feel a touch in new parts of their bodies. This unwillingness to move, of course, will prevent their ability to learn new skills. Therapeutic specialists should be able to make suggestions about how to help the child to tolerate the touch and desensitize him depending on the degree of defense. We hope that the physiotherapy and time will alleviate part of the sensory defense.

The child with Kabuki syndrome has a communication deficiency. After implementation of karate techniques, his social communication improved which was manifested in taking independent decisions, better interaction with other people, measured with VeeFim. Although we did not find another research in literature about Kabuki syndrome, the improvement in social communication due to implementation of karate techniques is confirmed from surveys on other disorders connected to communication problems. A survey by Bahrami et al. (2015) established that after 14-week karate trainings 30 children with autism spectrum disorders (ASD) had a significant reduction in their communication deficiency. This confirms the importance of adapted sports such as karate for achieving better motor coordination as well as better social functioning of children with different motor deficiencies.

\section{CONCLUSION}

The Kabuki syndrome is a rare genetic disorder and no definitive conclusions could be made.

The current study represents good practices for PT application in combination of adaptive sports. The results could be used as a basis for further in-depth research.

\section{REFERENCES}

Behrami, F., Movahedi, A.,Morandi, S., Sirensen, C. (2015). The Effect of Karate Techniques Training on Communication Deficit of Children with Autism Spectrum Disorders; Journal of Autism and Developmental Disorders , Springer; 46(3):978-86

Carcione, A., Piro, E., Albano, S. et al. (1991). Kabuki make-up (Niikawa-Kuroki) syndrome: Clinical and radiological observations in two sicilian children, Pediatric Radiology, Springer; 21: 428-431

Devriendt, K., Lemli, L., Craen, M., et al: (1995). Growth hormone deficiency and premature thelarche in a female infant with kabuki makeup syndrome. Hormon Resurch; 43:303-306.

Geneviève, D., et al. (2004). Atypical findings in Kabuki syndrome:Report of 8 patients in a series of 20 and review of the literature. Am J Med Genetics;129A:64-8

Halal, F., Gledhill, R., Dudkiewicz, A. (1989). Autosomal dominant inheritance of the Kabuki make-up (NiikawaKuroki) syndrome. Am J Med Genetics; 33(3):376-81

Hannibal, M., et al (2011). Spectrum of MLL2 (ALR) mutations in 110 cases of Kabuki syndrome. Am J Med Genetics; 155:1511-1516

Koutras, A., Fisher, S.(1982). Niikawa-Kuroki syndrome: A new malformation syndrome of postnatal dwarfism, 
mental retardation, unusual face, and protruding ears The Journal of Pediatrics, 101 (3) 417-419.

Kuroki, Y., Suzuki, Y., Chyo, H., Hata, A., Matsui, I. (1981). A new malformation syndrome of long palpebralfissures, large ears, depressed nasal tip, and skeletal anomalies associated with postnatal dwarfism and mental retardation; The Journal of Pediatrics, 99 (4) 570-573.

Niikawa, N., Kuroki, Y. (1982). The dermatoglyphic pattern of the Kabuki make-up syndrome Clinical Genetics; 121, (5); 315-320

Niikawa, N. et al. (1988). Kabuki make-up (NiikawaKuroki) syndrome: a study of 62 patients. Am J Med Genetics. 31(3):565-89

Matsumoto, N., Niikawa, N. (2003). Kabuki make-up syndrome: a review. Am J Med Genet C Semin Med Genet 117:57-65

Philip, N., et al. (1992). Kabuki make-up (NiikawaKuroki) syndrome: a study of 16 non-Japanese cases. Clin Dysmorphology;1(2):63-77.

Rouffiange, L., Dusabe, J., Docquier, P. (2012). Recurrent dislocation of the patella in kabuki make-up syndrome. Case Rep Orthopedy; 501453.

Russell, D., Rosenbaum, P., Avery, L., Lane, M. (2002).

Gross Motor Function Measure (GMFM-66 and GMFM-88) User's Manual. London, United Kingdom: Mac Keith Press;

Schott, D., Gerver, W., Stumpel, C. (2017). Growth Hormone Therapy in Children with Kabuki Syndrome: 1-year Treatment Results, Horm Res Paediatr; 88:258264.

Wada, A., Nakamura, T., Yamaguchi, T., et al. (2012). Surgical treatment of hip dislocation in Kabuki syndrome: use of incomplete periacetabular osteotomy for posterior acetabular wall deficiency. J Child Orthop.; 6(4):261-7.

\section{Corresponding author: Prof. Nezabravka Gencheva, PhD,} Departament Kinesitherapy and Rehabilitation, National Sports Academy "Vassil Levski", Sofia, Bulgaria E-mail: nezig@mail.bg 